\title{
Prediction of the molecular mechanisms and potential therapeutic targets for diabetic nephropathy by bioinformatics methods
}

\author{
WAN-NING WANG ${ }^{1}$, WEN-LONG ZHANG ${ }^{2}$, GUANG-YU ZHOU ${ }^{3}$, \\ FU-ZHE MA ${ }^{1}$, TAO SUN ${ }^{1}$, SEN-SEN SU ${ }^{1}$ and ZHONG-GAO XU ${ }^{1}$ \\ ${ }^{1}$ Department of Nephrology, The First Hospital of Jilin University, Changchun, Jilin 130021; Departments of ${ }^{2}$ Hematology \\ and Oncology, and ${ }^{3}$ Nephrology, China-Japan Union Hospital of Jilin University, Changchun, Jilin 130033, P.R. China
}

Received August 31, 2015; Accepted February 26, 2016

DOI: $10.3892 /$ ijmm.2016.2527

\begin{abstract}
In this study, we aimed to explore the molecular mechanisms of and genetic factors influencing diabetic nephropathy (DN). Gene expression profiles associated with DN were obtained from the GEO database (Accession no. GSE20844). The differentially expressed genes (DEGs) between diabetic mice and non-diabetic mice were screened. Subsequently, the DEGs were subjected to functional and pathway analysis. The protein-protein interaction (PPI) network was constructed and the transcription factors (TFs) were screened among the DEGs. A total of 92 upregulated and 118 downregulated genes were screened. Pathway analysis revealed that the p53 signaling pathway, the transforming growth factor (TGF) $-\beta$ signaling pathway and the mitogen-activated protein kinase (MAPK) signaling pathway were significantly enriched by upregulated genes. Serpine1 (also known as plasminogen activator inhibitor-1), early growth response 1 (Egrl) and Mdk were found to be significant nodes in the PPI network by three methods. A total of 12 TFs were found to be differentially expressed, of which nuclear receptor subfamily 4 , group A, member $1(\mathrm{Nr} 4 a \mathrm{l})$ and peroxisome proliferator-activated receptor gamma (Pparg) were found to have multiple interactions with other DEGs. We demonstrated that the p53 signaling pathway, the TGF- $\beta$ signaling pathway and the MAPK signaling pathway were dysregulated in the diabetic mice. The significant nodes (Serpine1, Egr1 and Mdk) and differentially expressed TFs (Nr4al and Pparg) may provide a novel avenue for the targeted therapy of DN.
\end{abstract}

\section{Introduction}

Diabetic nephropathy (DN) is a chronic kidney disease and is a serious complication of long-term diabetes mellitus (1).

Correspondence to: Dr Zhong-Gao Xu, Department of Nephrology, The First Hospital of Jilin University, 71 Xinmin Street, Changchun, Jilin 130021, P.R. China

E-mail: renalxu@163.com

Key words: molecular mechanism, therapeutic target, diabetic nephropathy, protein-protein interaction, pathways, transcription factor
DN develops in 30-40\% of patients with type I and II diabetes mellitus and is a risk factor for increased mortality in patients with cardiovascular disease (2). Although the signs of early DN are not evident, the clinical evidence for DN is the presence of proteinuria, glomerular hypertrophy, decreased glomerular filtration and a decline in renal function (1). DN continues to present a health concern worldwide.

Accumulating experimental in vivo and in vitro evidence has indicated that multiple pathways and cytokines play a role in the pathogenesis of DN. For example, a recent study suggested a cardinal role of inflammatory molecular and pathways in the pathogenesis of DN (3). The activation of the innate immune response associated with various inflammatory molecules, such as interleukin (IL)-1, IL-18 and tumor necrosis factor (TNF) has also been shown to contribute to the renal injury observed in patients with DN (4). Furthermore, it has been reported that the nuclear factor (NF)- $\mathrm{B}$ signaling pathway induces the expression of inflammatory genes during the progression of DN, and these effects are modulated by the Ras homolog gene family, member A (RhoA)/Rho-associated protein kinase (ROCK) signaling pathway (5). A good understanding of the molecular mechanisms responsible for the disease may aid in the development of effective therapies. However, the molecular mechanisms of DN have not yet been fully clarified.

Microarray data have been widely used to connect genes and molecules to diseases (6). Reiniger et al proved the target role of receptor for advanced glycation end-products (RAGE) in the treatment of DN based on microarray data (Accession no. GSE20844)(7). In the present study, we downloaded the same microarray data from the Gene Expression Omnibus (GEO) database. Subsequently, based on the gene expression profiles, the differentially expressed genes (DEGs) were analyzed and the DEG-related functions and pathways were predicted. The aim of the present study was to elucidate the mechanisms of DN pathogenesis and to identify associated significant genes.

\section{Data collection methods}

Data acquisition and preprocessing. Whole-genome microarray gene expression data for glomeruli from diabetic male OVE26 mice (diabetic group, $n=4$ ) and glomeruli from nondiabetic male FVB mice (control group, $n=3$ ) have been deposited in the GEO archive database (Accession no. GSE20844) (7). 

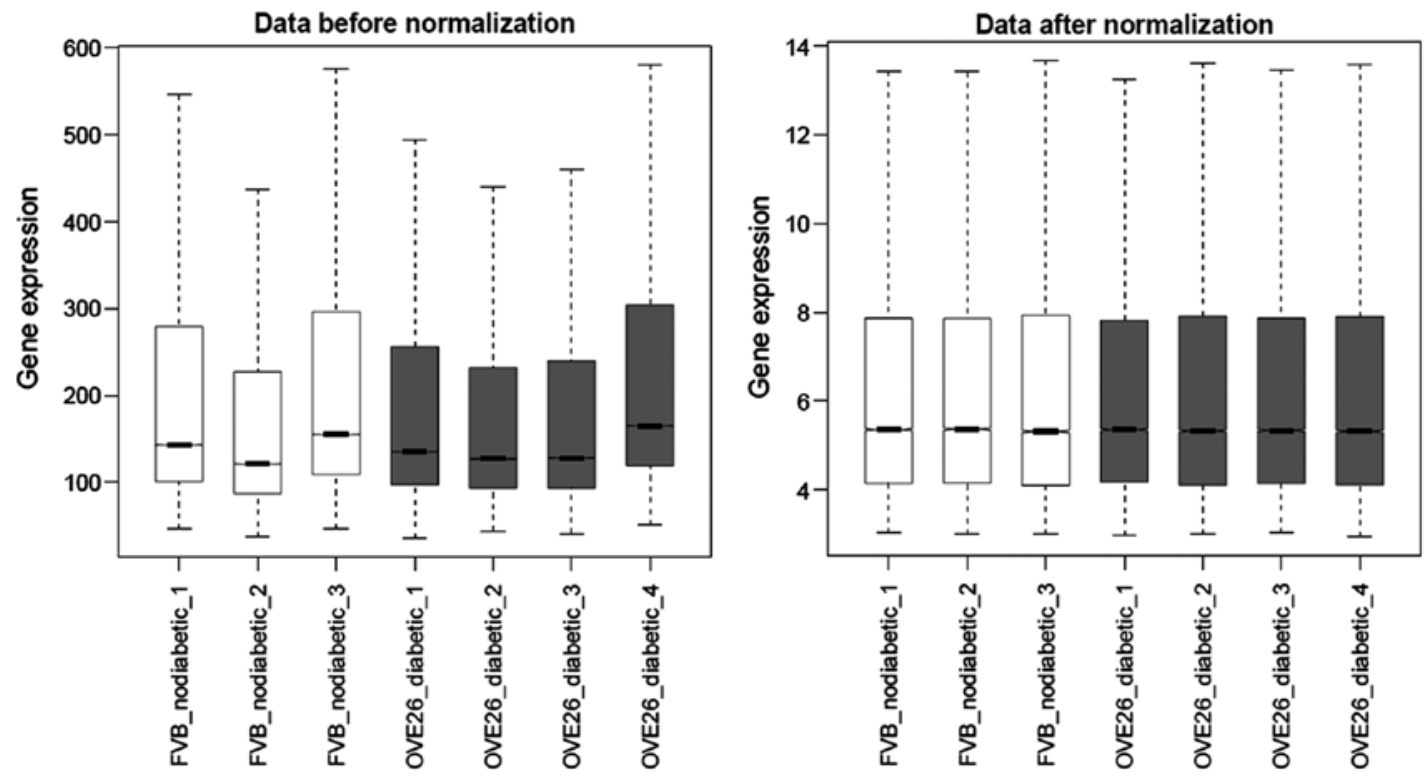

Figure 1. Gene expression data before and after normalization. Horizontal axis represents the sample symbol and the vertical axis represents the gene expression values. The black line in the box plot represents the median value of gene expression.

We downloaded the raw Affymetrix CEL files based on the platform of Affymetrix Mouse Genome 4302.0 Array.

The raw data underwent pre-processing, including background correction, quantile normalization and probe summarization with the application of bioconductor package 'affy', as previousy described (8).

Analysis of DEGs. The DEGs in the diabetic group compared with the non-diabetic controls were analyzed using the Bioconductor package 'limma', as previousy described (9). The P-value for each gene was calculated using the Student's t-test. Genes with differences in expression denoted by values of $\mathrm{p}<0.05$ and $\log _{2} \mathrm{FC}$ (fold change) $1 \geq 0.58$, screened as DEGs. In order to compare the differences in the profiles of DEGs between the diabetic and control samples, the gene expression data was clustered using R gplots software package (http://cran.r-project. org/web/packages/gplots/index.html.). Subsequently, the chromosomal location of the DEGs was explored based on the chip annotation information.

Gene Ontology (GO) and pathway analysis. The upregulated and downregulated genes were subjected to GO and Kyoto Encyclopedia of Genes and Genomes (KEGG) pathway analysis. The genes were enriched in three GO categories, such as biological process (BP), molecular function (MF) and cellular component (CC). The enrichment analysis based on the hypergeometric distribution was performed using the Database for Annotation, Visualization and Integrated Discovery (DAVID) online tool (10). The cut-off value for a significant GO term and pathway was set to $\mathrm{P}<0.05$ and count $\geq 2$.

Protein-protein interaction (PPI) network. The functional protein interactions among the DEGs and the encoding proteins were predicted using the Search Tool for the Retrieval of Interacting Genes (STRING) (11). The PPI score was set as 0.4 and other parameters were set as the default value. Cytoscape was used to visualize the PPI network.
Subsequently, with the application of CytoNCA (12), the hub nodes were measured based on the degree centrality (13), betweenness centrality (14) and subgraph centrality (15). The hub genes were then subjected to cluster analysis using the $\mathrm{R}$ gplots software package.

Transcription factor (TF) analysis. TFs encoded by DEGs were explored combined with the mouse TF information recorded in The Animal Transcription Factor DataBase (AnimalTFDB) (http://www.bioguo.org/AnimalTFDB/index.php) (16). Based on the TRANSFAC database, the TF-DEG interactions were predicted according to the information provided in the TRANSFAC database using the cytoscape plugin termed iRegulon (17).

\section{Results}

Identification of DEGs. After preprocessing, the gene expression data were normalized (Fig. 1). According to values of $\mathrm{p}<0.05$ and $\log _{2} \mathrm{FC}$ (fold change) $\mid \geq 0.58$, a total of 210 genes were found to be differentially expressed in the diabetic group, including 92 upregulated and 118 downregulated genes. Hierarchical clustering analysis revealed that the upregulated and downregulated genes were relatively distinguished between the different groups (Fig. 2).

As shown in Fig. 3, the majority of upregulated genes were located on chromosome 3 , whereas chromosomes 9 and 11 had the majority of downregulated genes.

GO and pathway analysis. The over-represented GO terms for the upregulated and downregulated genes are listed in Table I. Significantly enriched GO terms for upregulated genes included the regulation of angiogenesis, extracellular region and vascular endothelial growth factor receptor binding. For genes that were downregulated, the significantly enriched GO terms included cellular ion homeostasis, apical plasma membrane and symporter activity. 


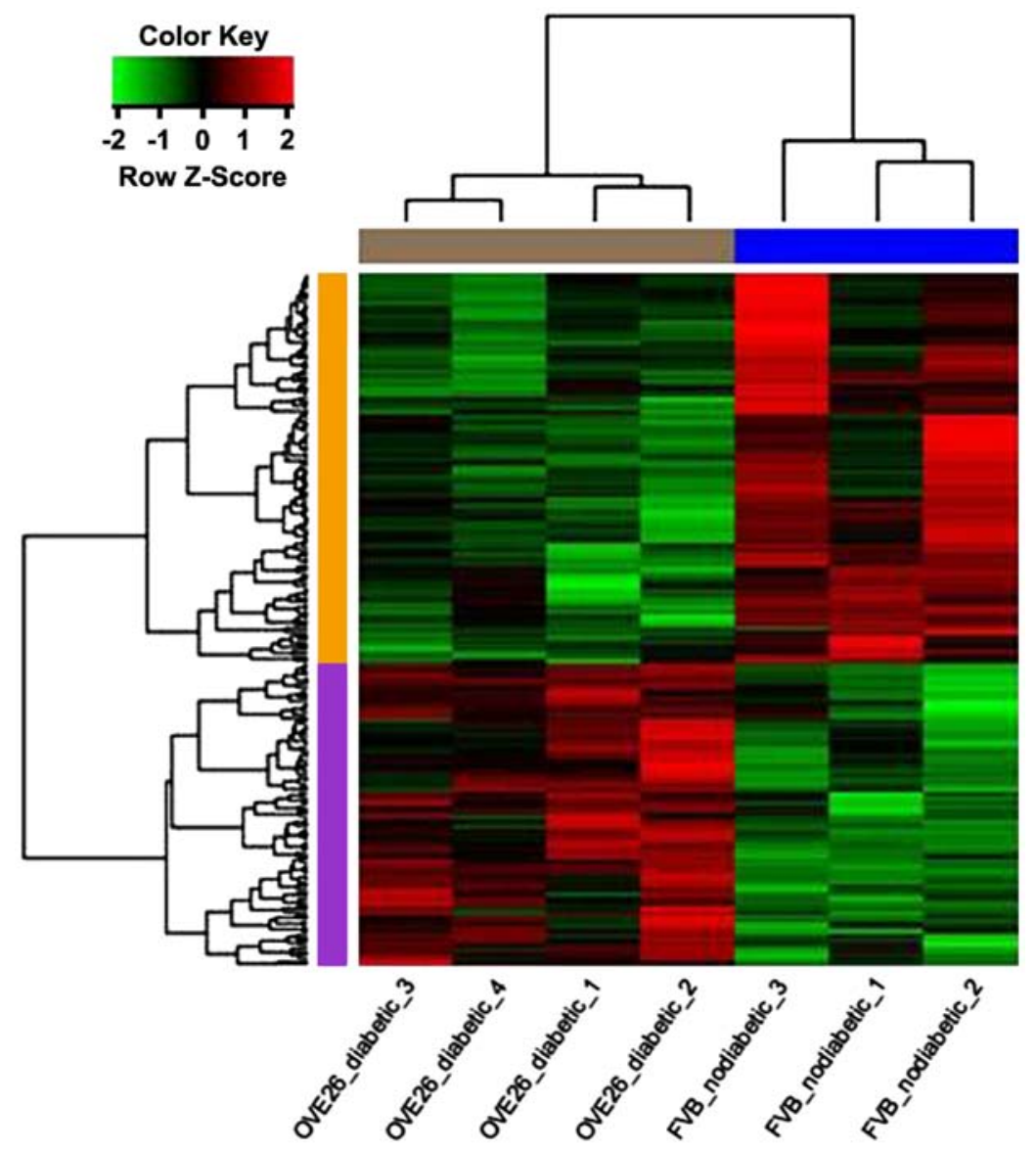

Figure 2. The heatmap of gene expression profiles in diabetic and non-diabetic samples. Green, low gene expression value; red, high gene expression value; black, no differential expression.
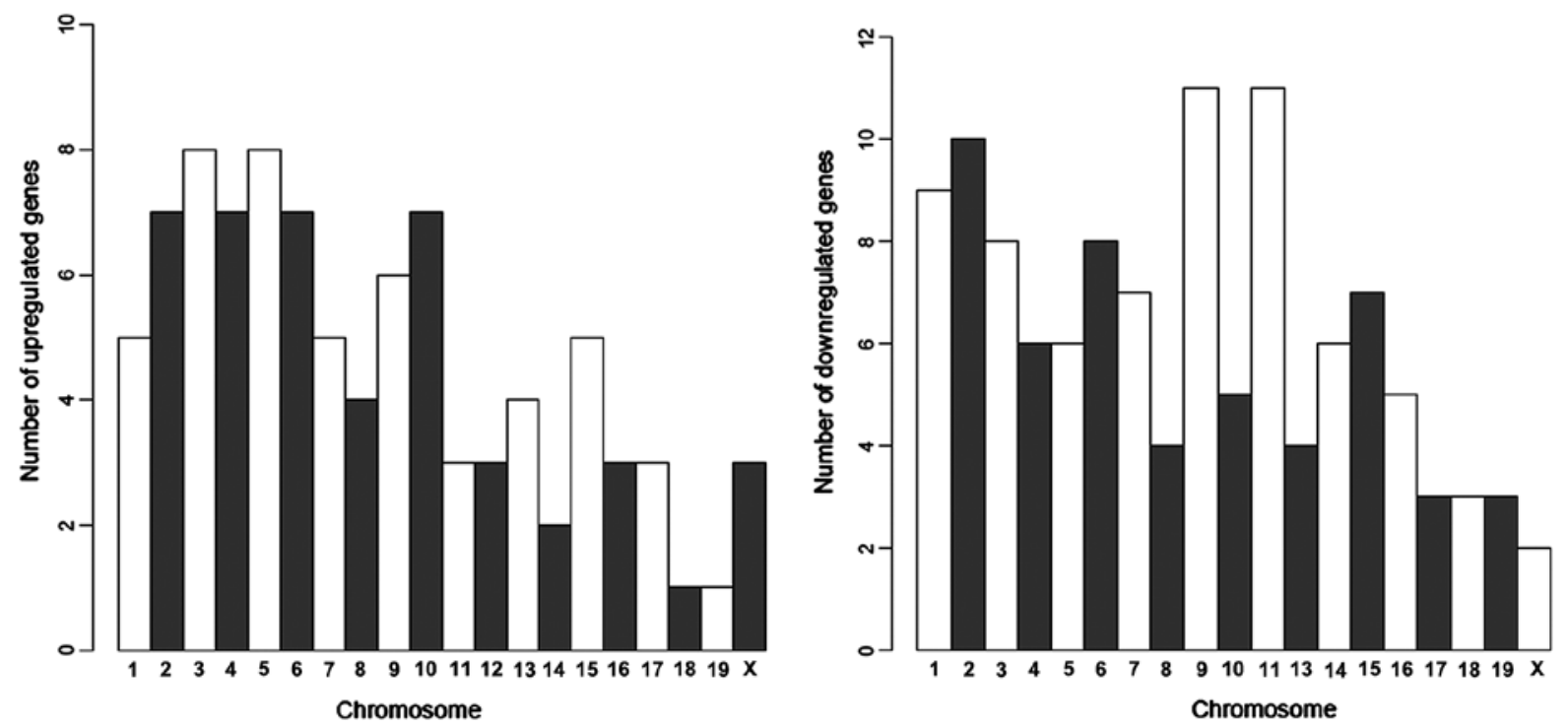

Figure 3. Distribution information of the upregulated and downregulated genes on the respective chromosomes.

Of the 7 significantly enriched pathways, Pentose and glucuronate interconversions were found to be closely associated with the downregulated genes. Other pathways, such as the p53 signaling pathway, cytokine-cytokine receptor interaction, the transforming growth factor (TGF) $-\beta$ signaling pathway and the mitogen-activated protein kinase (MAPK) signaling pathway were significantly enriched by upregulated genes (Table II).

PPI network. With a PPI score $>0.4$, a PPI network with 123 nodes and 219 edges was constructed, as shown in Fig. 4. The top 15 nodes based on the degree, betweenness and 
Table I. The significant GO terms enriched by DEGs.

\begin{tabular}{|c|c|c|}
\hline Term & Count & P-value \\
\hline \multicolumn{3}{|l|}{ Upregulated genes } \\
\hline \multicolumn{3}{|l|}{ BP } \\
\hline GO:0042127 - regulation of cell proliferation & 13 & $2.30 \mathrm{E}-05$ \\
\hline GO:0045765 - regulation of angiogenesis & 5 & $9.16 \mathrm{E}-05$ \\
\hline GO:0016525 - negative regulation of angiogenesis & 4 & $2.04 \mathrm{E}-04$ \\
\hline GO:0008285 - negative regulation of cell proliferation & 7 & 0.001184688 \\
\hline GO:0009611 - response to wounding & 8 & 0.002365745 \\
\hline \multicolumn{3}{|l|}{$\mathrm{CC}$} \\
\hline GO:0005576 - extracellular region & 20 & $5.60 \mathrm{E}-05$ \\
\hline GO:0044421 - extracellular region part & 11 & 0.001793488 \\
\hline GO:0046658 - anchored to plasma membrane & 3 & 0.002476471 \\
\hline GO:0005615 - extracellular space & 8 & 0.006777968 \\
\hline GO:0044459 - plasma membrane part & 14 & 0.022475418 \\
\hline \multicolumn{3}{|l|}{ MF } \\
\hline GO:0005172 - vascular endothelial growth factor receptor binding & 3 & $2.41 \mathrm{E}-04$ \\
\hline GO:0005539 - glycosaminoglycan binding & 5 & 0.002459879 \\
\hline GO:0030247 - polysaccharide binding & 5 & 0.00373434 \\
\hline GO:0001871 - pattern binding & 5 & 0.00373434 \\
\hline GO:0030246 - carbohydrate binding & 7 & 0.00480543 \\
\hline \multicolumn{3}{|l|}{ Downregulated genes } \\
\hline \multicolumn{3}{|l|}{$\mathrm{BP}$} \\
\hline GO:0006873 - cellular ion homeostasis & 7 & 0.002754032 \\
\hline GO:0055082 - cellular chemical homeostasis & 7 & 0.003139335 \\
\hline GO:0048878 - chemical homeostasis & 8 & 0.003390517 \\
\hline GO:0050801 - ion homeostasis & 7 & 0.004850765 \\
\hline GO:0019725 - cellular homeostasis & 7 & 0.010204447 \\
\hline \multicolumn{3}{|l|}{$\mathrm{CC}$} \\
\hline GO:0016324 - apical plasma membrane & 6 & $3.76 \mathrm{E}-04$ \\
\hline GO:0005576 - extracellular region & 22 & 0.001139987 \\
\hline GO:0045177 - apical part of cell & 6 & 0.001580783 \\
\hline GO:0005615 - extracellular space & 9 & 0.014078693 \\
\hline GO:0005903 - brush border & 3 & 0.020010741 \\
\hline \multicolumn{3}{|l|}{ MF } \\
\hline GO:0015293 - symporter activity & 5 & 0.008223849 \\
\hline GO:0019807 - aspartoacylase activity & 2 & 0.012005144 \\
\hline GO:0008201 - heparin binding & 4 & 0.013672168 \\
\hline GO:0004046 - aminoacylase activity & 2 & 0.023867944 \\
\hline GO:0031402 - sodium ion binding & 4 & 0.024166754 \\
\hline
\end{tabular}

GO, Gene Ontology; DEGs, differentially expressed genes; BP, biological process; CC, cellular component; MF, molecular function.

subgraph centralities were screened (Table III). Top 3 nodes such as early growth response 1 (Egr1), Serpine1 [also known as plasminogen activator inhibitor-1 (PAI-1)] and Mdk were shared based on the degree, betweenness and subgraph centralities. Merging the overlapping genes, we obtained 24 significant genes. Hierarchical clustering analysis revealed that the diabetic and non-diabetic samples were distinguished based on the gene expression profiles of the 24 significant genes (Fig. 5), suggesting that these genes were feature genes in diabetic samples.

In order to analyze the pathways associated with these feature genes, we performed KEGG pathway analysis. As shown in Table IV, the significant genes were closely associated with the MAPK signaling pathway, the p53 signaling pathway and the TGF- $\beta$ signaling pathway.

Analysis of TFs. Combined with the TF information recorded in TFDB, we obtained 12 differentially expressed TFs from 9 TF families (Table V). The interactions between differentially expressed TFs and DEGs predicted by the TRANSFAC database are shown in Fig. 6. The TFs, nuclear receptor subfamily 4, group A, member 1 ( Nr4a1) and peroxisome proliferator-activated receptor gamma (Pparg), were shown to have interactions with multiple genes. 


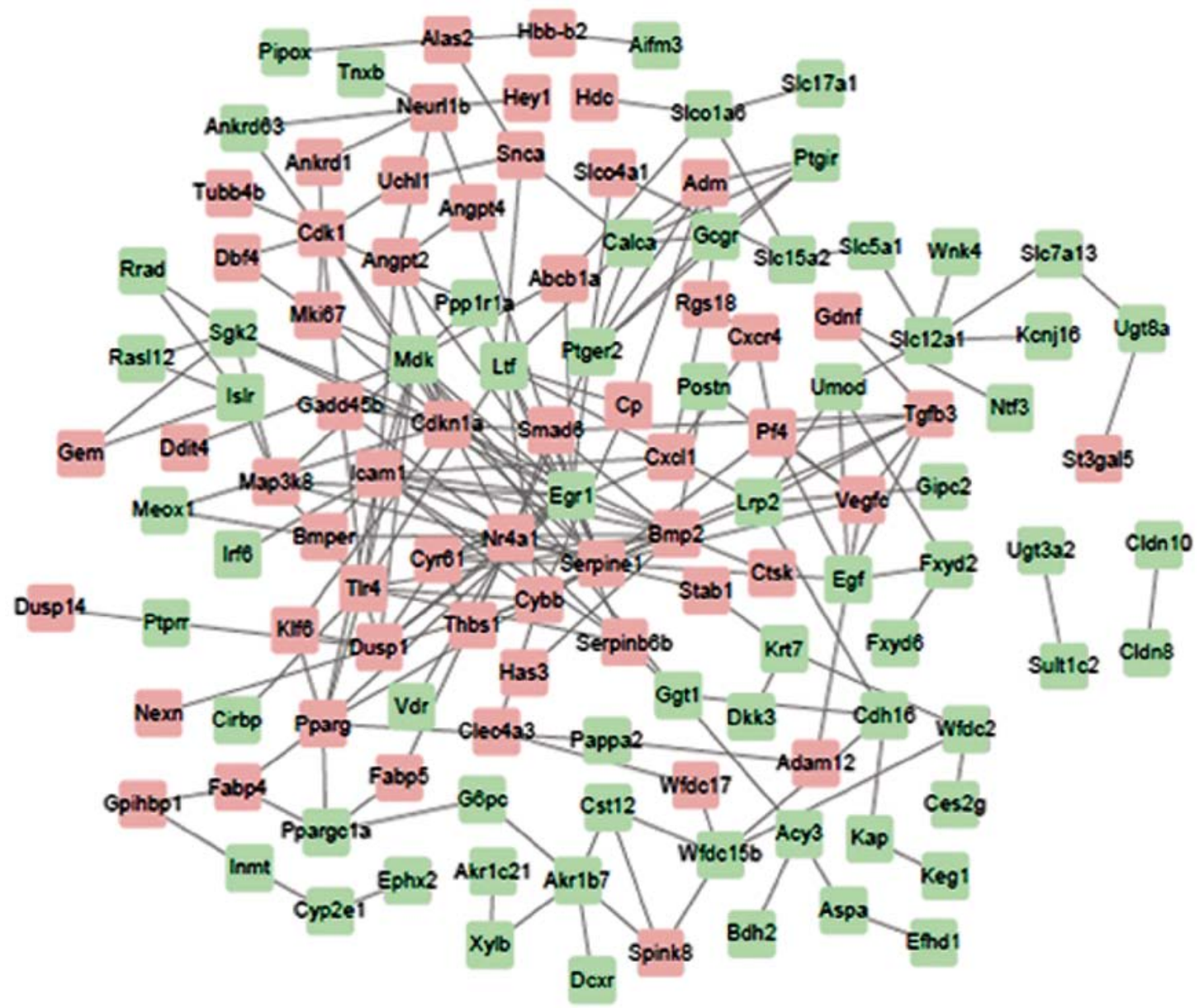

Figure 4. Protein-protein interaction (PPI) network for differentially expressed genes (DEGs). Green, downregulated genes; red, upregulated genes.

Table II. Pathways significantly enriched by differentially expressed genes.

\begin{tabular}{lcc}
\hline Term & Count & P-value \\
\hline Upregulation & & \\
mmu04115: p53 signaling pathway & 5 & $7.57 \mathrm{E}-04$ \\
mmu04110: Cell cycle & 5 & 0.007243672 \\
mmu04060: Cytokine-cytokine & 6 & 0.015248477 \\
receptor interaction & & \\
mmu04350: TGF- $\beta$ signaling pathway & 4 & 0.015533727 \\
mmu04010: MAPK signaling pathway & 6 & 0.021089527 \\
mmu05219: Bladder cancer & 3 & 0.026718766 \\
Downregulation & & \\
$\quad$ mmu00040: Pentose and glucuronate & 3 & 0.003652816 \\
interconversions & & \\
\hline
\end{tabular}

\section{Discussion}

DN is a chronic kidney disease and is more prevalent in patients with diabetes mellitus (18). ND increases the risk factor of cardiovascular disease and mortality in diabetic patients (19). There is thus a need for the development of more effective treatments for patients with DN. In this study, we attempted to explore the potential molecular mechanisms of DN based on the bioinformatics methods and to provide a prospective novel therapeutic target. In the present study, we screened out 92 upregulated and 118 downregulated genes. All the genes with changes in expression were proven to be significant according to hierarchical clustering analysis.

KEGG pathway analysis for both DEGs and significant nodes in the PPI network revealed that the p53 signaling pathway, the TGF- $\beta$ signaling pathway and the MAPK signaling pathway were the significantly enriched pathways. It has been reported that the overexpression of p53 is associated with the progression of DN. The expression of p53 and TGF- $\beta$ was shown to be overexpressed in the renal cortex of diabetic mice. The crosstalk between p53 and miR-192, which is mediated by TGF- $\beta$ was shown to be involved in the pathogenesis of DN (20). The MAPK signaling pathway is a regulator of the expression of pro-inflammatory molecules in DN. Targeted therapy for inhibiting the p38 MAPK signaling pathway has shown preventive effects on streptozotocin-induced DN (21). The p38 MAPK signaling pathway also plays a partial role in fibrosis associated with DN (22). These findings suggest that our findings are significant.

In our study, the PPI network showed that Egr1, Serpine1 and Mdk were the top 3 nodes based on the degree of centrality, betweenness centrality and subgraph centrality. Serpine1, also known as PAI-1, is a serine protease inhibitor and a key regulator of extracellular matrix (ECM). PAI-1 
Table III. Top 15 significant nodes in the protein-protein interaction network based on degree, betweenness and subgraph centralities.

\begin{tabular}{|c|c|c|c|c|c|}
\hline Node & Degree & Node betweenness & Betweenness & Node subgragh & Subgragh \\
\hline Serpine1 & 16 & Serpine1 & 2905.323 & Egr1 & 337.665 \\
\hline Egr1 & 15 & Egr1 & 2433.539 & Serpine1 & 337.1419 \\
\hline Mdk & 14 & Mdk & 2319.191 & Mdk & 285.9198 \\
\hline Nr4a1 & 12 & Pparg & 2105.681 & Icam1 & 209.8053 \\
\hline Icam1 & 12 & Lrp2 & 2064.846 & Cdkn1a & 187.7399 \\
\hline Cdkn1a & 12 & Cdh16 & 1583.724 & Nr4a1 & 178.7159 \\
\hline Tlr4 & 11 & Umod & 1440.558 & Tlr4 & 147.8937 \\
\hline Cdk1 & 10 & Ltf & 1421.087 & Dusp1 & 119.8608 \\
\hline Bmp2 & 10 & Ggt1 & 1384.699 & Bmp2 & 117.067 \\
\hline Dusp1 & 9 & Tlr4 & 1286.763 & Cyr61 & 104.8729 \\
\hline Pparg & 8 & Slc12a1 & 1250.098 & Pparg & 85.67106 \\
\hline Ltf & 7 & Nr4a1 & 1248.253 & Cybb & 68.2394 \\
\hline Egf & 7 & Icam1 & 1223.037 & Smad6 & 63.11027 \\
\hline Cybb & 7 & Cdk1 & 1119.84 & Cdk1 & 56.22912 \\
\hline Tgfb3 & 7 & Wfdc15b & 1082.356 & Map3k8 & 52.32016 \\
\hline
\end{tabular}

Table IV. The significant pathways enriched by 24 significant nodes in the protein-protein interaction network.

\begin{tabular}{lll}
\hline Term & P-value & \multicolumn{1}{c}{ Genes } \\
\hline mmu04010: MAPK signaling pathway & 0.005210429 & Dusp1, Map3K8,Tgfb3,Nr4A1, Egf \\
mmu05200: Pathways in cancer & 0.010457331 & Cdkn1A, Bmp2, Pparg, Tgfb3,Egf \\
mmu04115: p53 signaling pathway & 0.015340116 & Cdk1, Cdkn1A, Serpine1 \\
mmu04350: TGF- $\beta$ signaling pathway & 0.023759324 & Bmp2, Smad6,Tgfb3 \\
mmu04110: Cell cycle & 0.048314969 & $C d k 1, C d k n 1 A, T g f b 3$ \\
\hline
\end{tabular}

Table V. Differentially expressed transcription factors in diabetic samples.

\begin{tabular}{ll}
\hline TF family & TF gene symbol \\
\hline bHLH & Atoh8 \\
bHLH & Heyl \\
HMG & Tox2 \\
Homeobox & Meoxl \\
Homeobox & Six4 \\
IRF & Irf6 \\
MH1 & Smad6 \\
Nuclear orphan receptor & Nr4al \\
PPAR receptor & Pparg \\
Thyroid hormone receptor & Vdr \\
zf-C2H2 & Klf6 \\
zf-C2H2 & Egrl \\
\hline
\end{tabular}

has been widely investigated in many diseases including the kidney disease $(23,24)$. It is reported that ECM accumulation is implicated in the development and progression of DN (25). The expression of PAI-1 contributes to the fibrosis of kidney by inhibiting ECM degradation (26). PAI-1 has been proposed to be the potential target in renal fibrogenesis (26). PAI-1 was found to be overexpressed in the kidney of diabetic mice and its deficiency prevents glomerular injury of diabetic mice (27). Therefore, PAI-1 contributes to the progression of DN and PAI-1 knockdown may prove to be an effective therapeutic strategy for the treatment of DN. Additionally, midkine encoded by the $M d k$ gene has been proven to play a physiological role in kidney disease, including DN. $M d k$ plays a role in the occurrence and progression of acute kidney injury and contributes to the development of DN (28). Recent evidence has indicated that the diverse role of $M d k$ may open a new avenues for targeted therapies for DN (28).

Furthermore, Egrl is a TF and plays a role in inducing the overexpression of heparanase in DN. The upregulation of heparanase is closely associated with albuminuria and renal damage in diabetic mice (29). The inhibition of Egrl may be an effective strategy for preventing DN in diabetes. In the present study, other TFs, such as Nr4al and Pparg were found to play regulatory roles in the differential expression of genes. $\mathrm{Nr} 4 \mathrm{al}$ is a member of the nuclear orphan receptor family of TFs. Nr4a1 has been found to play a significant role in atherosclerosis, psoriasis and other chronic inflammatory diseases (30). As we all know, hypertension is closely related with the progression of DN. Nr4al has been found to be differentially expressed in the kidneys of hypertensive patients (31). Nr4al is a susceptible 


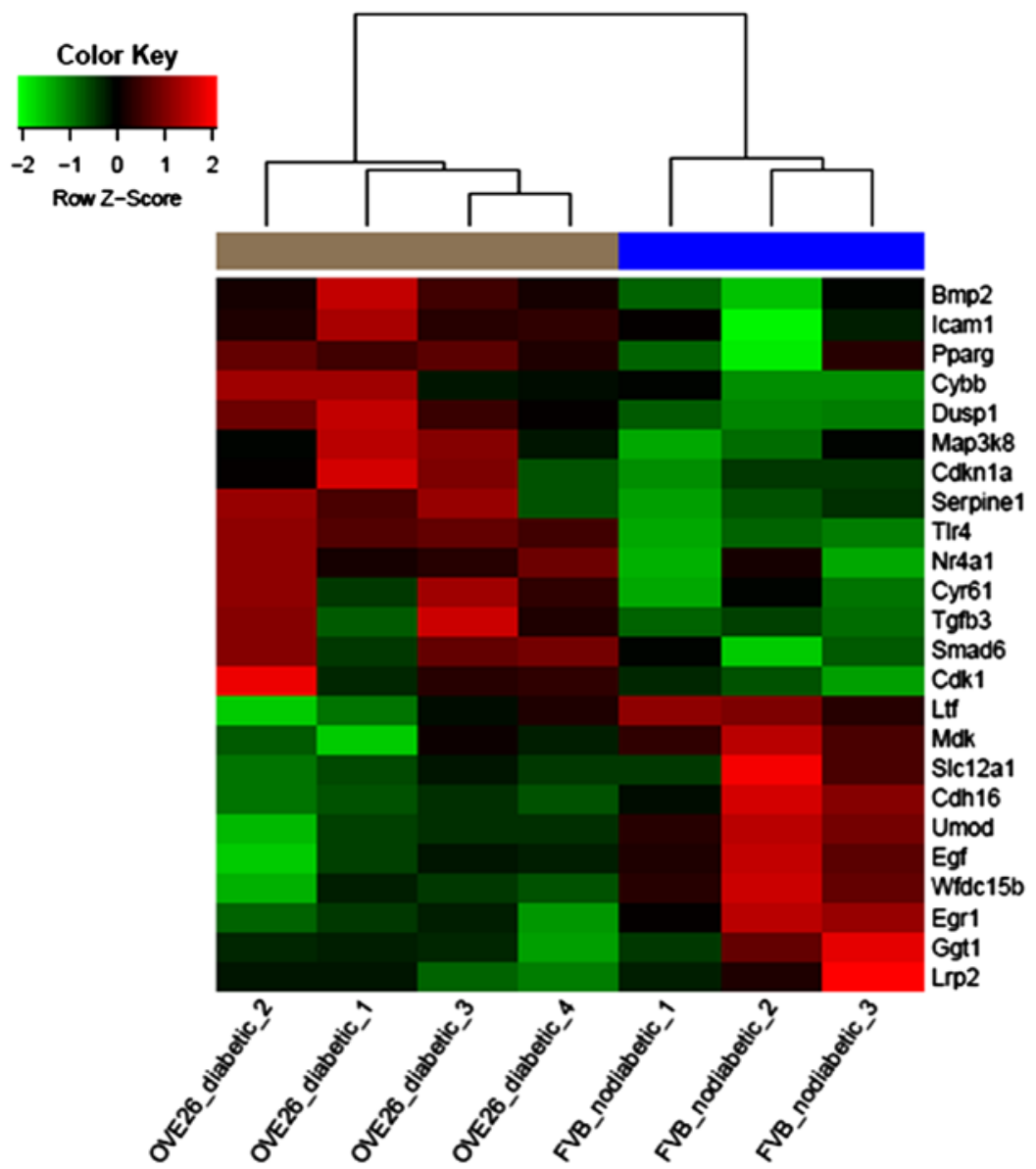

Figure 5. Hierarchical clustering analysis for the gene expression profiles of 24 significant genes in the protein-protein interaction (PPI) network.

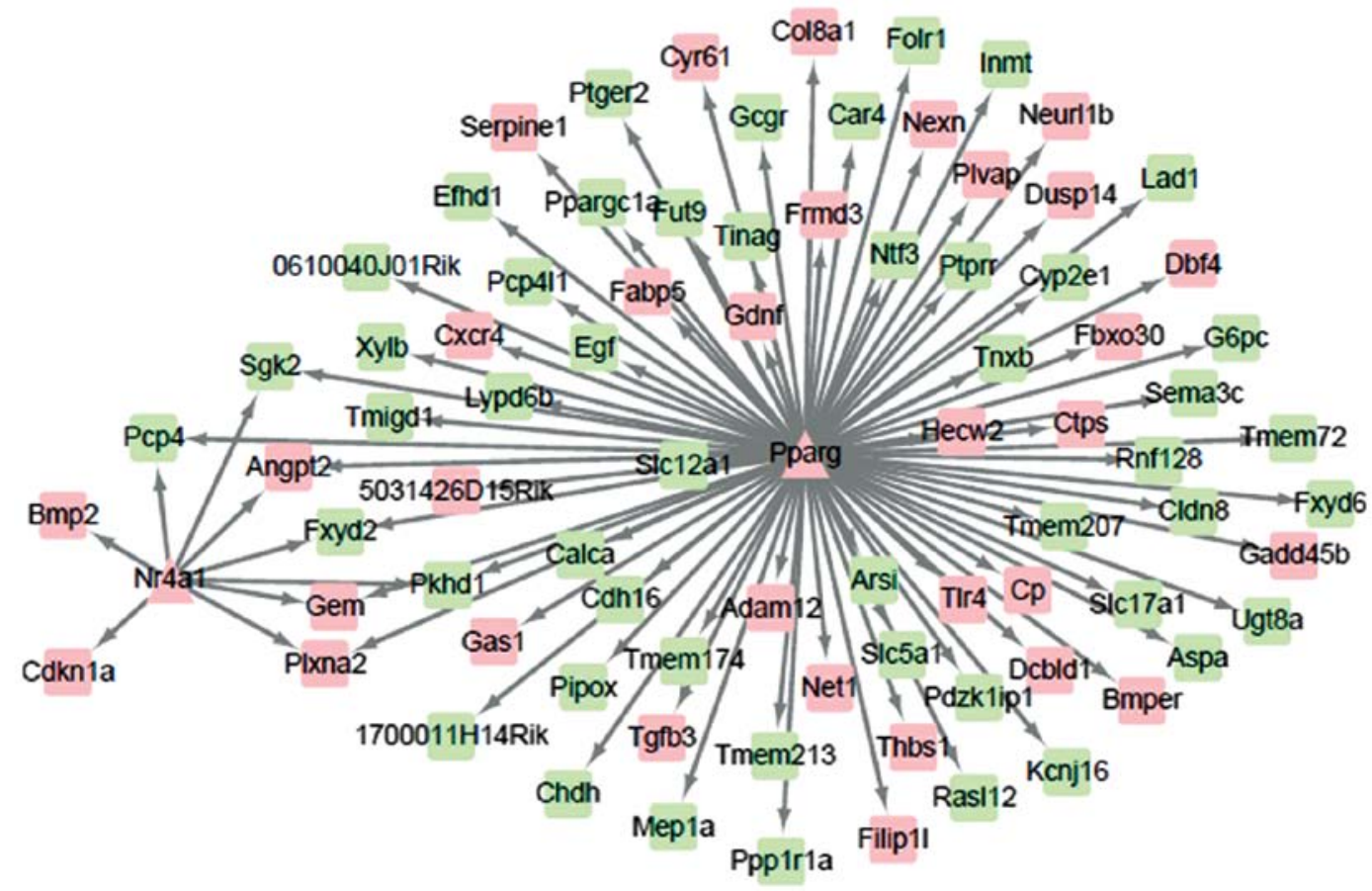

Figure 6. Interactions between transcription factors (TFs) and target genes. Red, upregulated genes; green, downregulated genes. Triangle represents TFs and the box plot represents target genes.

gene in chronic kidney disease and a deficiency in Nr4al has been shown to be involved in kidney injury and renal func- tion disorder (32). Otherwise, the other TF, Pparg, from the TF PPAR receptor family has been found to be associated with 
DN. Previous studies have demonstrated that Pparg variation contributes to DN development in type 2 diabetes (33). Thus, the significant genes identified in our study may be candidate therapeutic targets in DN.

Although we predicted the significant genes and pathways involved in the pathogenesis of DN, the lack of experimental validation was a limitation in our study. Experimental studies need be conducted to validate the differential gene expression profile and gene interaction pairs in the future.

In conclusion, we proved the significant role of the p53 signaling pathway, the TGF- $\beta$ signaling pathway and the MAPK signaling pathway in the progression of DN. The significant genes, such as Egrl, Serpine1, Mdk, Nr4al and Pparg may prove to be potential therapeutic targets for the treatment of DN. Our findings need be further validated by experimental evidence.

\section{Acknowledgements}

The project was supported by grants from the National Natural Science Foundation of China (nos. 81070578 and 81270809).

\section{References}

1. Sun YM, Su Y, Li J and Wang LF: Recent advances in understanding the biochemical and molecular mechanism of diabetic nephropathy. Biochem Biophys Res Commun 433: 359-361, 2013

2. Gross JL, de Azevedo MJ, Silveiro SP, Canani LH, Caramori ML and Zelmanovitz T: Diabetic nephropathy: diagnosis, prevention, and treatment. Diabetes Care 28: 164-176, 2005.

3. Navarro-González JF, Mora-Fernández C, Muros de Fuentes M and García-Pérez J: Inflammatory molecules and pathways in the pathogenesis of diabetic nephropathy. Nat Rev Nephrol 7: 327-340, 2011.

4. Luis-Rodríguez D, Martínez-Castelao A, Górriz JL, De-Álvaro F and Navarro-González JF: Pathophysiological role and therapeutic implications of inflammation in diabetic nephropathy World J Diabetes 3: 7-18, 2012.

5. Xie X, Peng J, Chang X, Huang K, Huang J, Wang S, Shen X, Liu P and Huang H: Activation of RhoA/ROCK regulates NF- $\kappa \mathrm{B}$ signaling pathway in experimental diabetic nephropathy. Mol Cell Endocrinol 369: 86-97, 2013.

6. Lamb J, Crawford ED, Peck D, Modell JW, Blat IC, Wrobel MJ, Lerner J, Brunet J-P, Subramanian A, Ross KN, et al: The Connectivity Map: using gene-expression signatures to connect small molecules, genes, and disease. Science 313: 1929-1935, 2006

7. Reiniger N, Lau K, McCalla D, Eby B, Cheng B, Lu Y, Qu W, Quadri N, Ananthakrishnan R, Furmansky M, et al: Deletion of the receptor for advanced glycation end products reduces glomerulosclerosis and preserves renal function in the diabetic OVE26 mouse. Diabetes 59: 2043-2054, 2010.

8. Gautier L, Cope L, Bolstad BM and Irizarry RA: affy-analysis of Affymetrix GeneChip data at the probe level. Bioinformatics 20: 307-315, 2004.

9. Smyth GK: limma: linear models for microarray data. In: Bioinformatics and Computational Biology Solutions Using $R$ and Bioconductor. Gentleman R, Carey VJ, Huber W, Irizarry RA and Dudoit S (eds). Springer, New York, NY, pp397-420, 2005.

10. Huang W, Sherman BT and Lempicki RA: Systematic and integrative analysis of large gene lists using DAVID bioinformatics resources. Nat Protoc 4: 44-57, 2009.

11. Szklarczyk D, Franceschini A, Wyder S, Forslund K, Heller D, Huerta-Cepas J, Simonovic M, Roth A, Santos A, Tsafou KP, et al: STRING v10: protein-protein interaction networks, integrated over the tree of life. Nucleic Acids Res 43: D447-D452, 2014

12. Tang Y, Li M, Wang J, Pan Y and Wu FX: CytoNCA: a cytoscape plugin for centrality analysis and evaluation of protein interaction networks. Biosystems 127: 67-72, 2015.

13. Jeong H, Mason SP, Barabási AL and Oltvai ZN: Lethality and centrality in protein networks. Nature 411: 41-42, 2001.
14. Goh KI, Oh E, Kahng B and Kim D: Betweenness centrality correlation in social networks. Phys Rev E Stat Nonlin Soft Matter Phys 67: 017101, 2003.

15. Estrada E and Rodríguez-Velázquez JA: Subgraph centrality in complex networks. Phys Rev E Stat Nonlin Soft Matter Phys 71: 056103, 2005.

16. Zhang HM, Liu T, Liu CJ, Song S, Zhang X, Liu W, Jia H, Xue Y and Guo AY: AnimalTFDB 2.0: a resource for expression, prediction and functional study of animal transcription factors. Nucleic Acids Res 43: D76-D81, 2014.

17. Janky R, Verfaillie A, Imrichová H, Van de Sande B, Standaert L, Christiaens V, Hulselmans G, Herten K, Naval Sanchez M, Potier D, et al: iRegulon: from a gene list to a gene regulatory network using large motif and track collections. PLoS Comput Biol 10: e1003731, 2014.

18. Go AS, Chertow GM, Fan D, McCulloch CE and Hsu CY: Chronic kidney disease and the risks of death, cardiovascular events, and hospitalization. N Engl J Med 351: 1296-1305, 2004.

19. Pfeffer MA, Burdmann EA, Chen C-Y, Cooper ME, de Zeeuw D, Eckardt K-U, Feyzi JM, Ivanovich P, Kewalramani R, Levey AS, et al; TREAT Investigators: A trial of darbepoetin alfa in type 2 diabetes and chronic kidney disease. N Engl J Med 361: 2019-2032, 2009.

20. Deshpande SD, Putta S, Wang M, Lai JY, Bitzer M, Nelson RG, Lanting LL, Kato $M$ and Natarajan R: Transforming growth factor- $\beta$-induced cross talk between p53 and a microRNA in the pathogenesis of diabetic nephropathy. Diabetes 62: 3151-3162, 2013.

21. Fang Y, Tian X, Bai S, Fan J, Hou W, Tong H and Li D: Autologous transplantation of adipose-derived mesenchymal stem cells ameliorates streptozotocin-induced diabetic nephropathy in rats by inhibiting oxidative stress, pro-inflammatory cytokines and the p38 MAPK signaling pathway. Int J Mol Med 30: 85-92, 2012.

22. Adhikary L, Chow F, Nikolic-Paterson DJ, Stambe C, Dowling J, Atkins RC and Tesch GH: Abnormal p38 mitogen-activated protein kinase signalling in human and experimental diabetic nephropathy. Diabetologia 47: 1210-1222, 2004.

23. Oda T, Jung YO, Kim HS, Cai X, López-Guisa JM, Ikeda Y and Eddy AA: PAI-1 deficiency attenuates the fibrogenic response to ureteral obstruction. Kidney Int 60: 587-596, 2001.

24. Kitching AR, Kong YZ, Huang XR, Davenport P, Edgtton KL, Carmeliet P, Holdsworth SR and Tipping PG: Plasminogen activator inhibitor-1 is a significant determinant of renal injury in experimental crescentic glomerulonephritis. J Am Soc Nephrol 14: 1487-1495, 2003.

25. Alvarez ML and DiStefano JK: Functional characterization of the plasmacytoma variant translocation 1 gene (PVT1) in diabetic nephropathy. PLoS One 6: e18671, 2011.

26. Rerolle JP, Hertig A, Nguyen G, Sraer JD and Rondeau EP: Plasminogen activator inhibitor type 1 is a potential target in renal fibrogenesis. Kidney Int 58: 1841-1850, 2000.

27. Lassila M,Fukami K, Jandeleit-Dahm K, Semple T, Carmeliet P, Cooper ME and Kitching AR: Plasminogen activator inhibitor-1 production is pathogenetic in experimental murine diabetic renal disease. Diabetologia 50: 1315-1326, 2007.

28. Kosugi T and Sato W: Midkine and the kidney: health and diseases. Nephrol Dial Transplant 27: 16-21, 2012.

29. Gil N, Goldberg R, Neuman T, Garsen M, Zcharia E, Rubinstein AM, van Kuppevelt T, Meirovitz A, Pisano C, Li JP, et al: Heparanase is essential for the development of diabetic nephropathy in mice. Diabetes 61: 208-216, 2012.

30. McMorrow JP and Murphy EP: Inflammation: a role for NR4A orphan nuclear receptors? Biochem Soc Trans 39: 688-693, 2011.

31. Hagiwara S, Mcclelland A and Kantharidis P: MicroRNA in diabetic nephropathy: renin angiotensin, aGE/RAGE, and oxidative stress pathway. J Diabetes Res 2013: 173783, 2013.

32. Westbrook L, Johnson AC, Regner KR, Williams JM, Mattson DL, Kyle PB, Henegar JR and Garrett MR: Genetic susceptibility and loss of Nr4a1 enhances macrophage-mediated renal injury in CKD. J Am Soc Nephrol 25: 2499-2510, 2014.

33. Lapice E, Pinelli M, Riccardi G and Vaccaro O: Pro12Ala polymorphism in the PPARG gene contributes to the development of diabetic nephropathy in Chinese type 2 diabetic patients: comment on the study Liu et al. Diabetes Care 33: e114, author reply e115, 2010. 